\title{
Deconvolution in Measurements of Muon Neutrino Energy Spectra with IceCube
}

\author{
Tim Ruhe ${ }^{1, *}$ for the IceCube Collaboration** \\ ${ }^{1}$ TU Dortmund University, Dortmund, Germany
}

\begin{abstract}
As the energy of an incident neutrino cannot be accessed experimentally, muon neutrino energy spectra have to be inferred from energy-dependent observables, using deconvolution algorithms. This paper discusses the challenges associated with the application of deconvolution algorithms and presents two examples of spectral measurements obtained using the IceCube neutrino telescope in the 59- and 79-string configuration.
\end{abstract}

\section{Neutrino Energy Spectra and Challenges in Deconvolution}

In IceCube muon-neutrinos are not detected directly, but via secondary muons produced in charged-current (CC) interactions of the incident neutrino with nuclei in the ice or the bedrock. The neutrino energy spectrum $\mathrm{d} N / \mathrm{d} E_{v}$ is therefore experimentally inaccessible and has to be inferred using the energy spectrum of secondary muons $\mathrm{d} N_{\mu} / \mathrm{d} E_{\mu}$. The production of a muon of energy $E_{\mu}$ from an incident neutrino of energy $E_{\nu}$ is a stochastical process and its chance probability is given as $\mathrm{dP}\left(\mathrm{E}_{\gamma}\right) / \mathrm{d} E_{\mu}$, which also contains details on the neutrinonucleon interaction. $\mathrm{d} N_{\mu} / \mathrm{d} E_{\mu}$ and $\mathrm{d} N_{v} / \mathrm{d} E_{\nu}$ are connected by the following equation [1], which mathematically corresponds to a Fredholm integral equation of the first kind:

$$
\frac{\mathrm{d} N_{\mu}}{\mathrm{d} E_{\mu}}=\int_{E_{\mu}}^{\infty}\left(\frac{\mathrm{d} N_{v}}{\mathrm{~d} E_{v}}\right)\left(\frac{\mathrm{dP}\left(\mathrm{E}_{v}\right)}{\mathrm{d} E_{\mu}}\right) \mathrm{d} E_{v} .
$$

The energy of the muon, however, cannot be accessed experimentally either, but has to be obtained either from energy-dependent observables, like the number of detected photons or length of the track or from the estimated energy of the muon. The finite resolution of these quantities, with respect to the muon energy and the limited acceptance of the detector further complicate the problem. Muons of the exact same energy, may create significantly different signatures in the detector, depending on whether they, for example, cut the detector close to a corner or traverse the entire instrumented volume. The $v_{\mu}$ energy spectrum therefore has to be obtained via a deconvolution procedure.

The overall spectrum of $v_{\mu}$ is expected to be a superposition of three components. The first component are so-called conventional atmospheric neutrinos, originating from the decay of pions and kaons produced in cosmic ray airshowers. Due to the relatively long lifetime of pions and kaons $\tau \approx 10^{-8} \mathrm{~s}[2]$, the spectrum of conventional atmospheric neutrinos is approximately one power steeper than the cosmic ray energy spectrum $\left(\mathrm{d} N_{\text {conv }} / \mathrm{d} E_{v} \approx E^{-3.7}\right)$.

\footnotetext{
*e-mail: tim.ruhe@tu-dortmund.de

***Authorlist and acknowledgement http://icecube.wisc.edu
} 


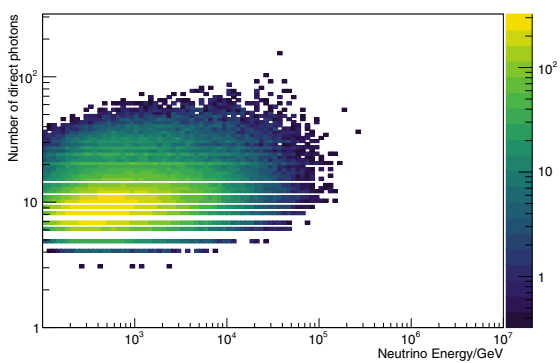

(a) Correlation of the number of photons with small time residuals with the energy of the neutrino.

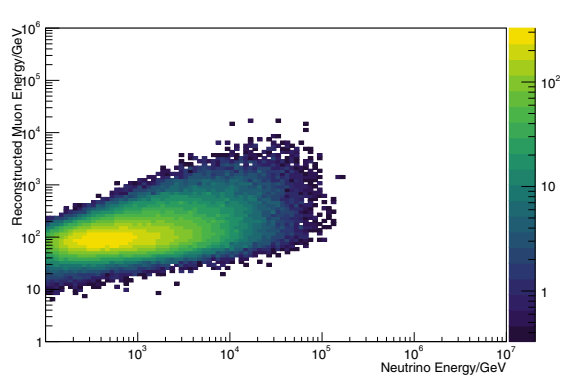

(b) Correlation of the reconstructed muon energy with the energy of the neutrino.

Figure 1. Correlation of the input variables with the true energy of the neutrino for the IC-79 analysis.

The second component are prompt atmospheric neutrinos, from the decay of charmed particles. Due to their short lifetime $\tau \approx 10^{-12} \mathrm{~s}$ [2], prompt atmospheric neutrinos inherit the cosmic ray spectrum more directly and the resulting flux is $\mathrm{d} N_{\text {prompt }} / \mathrm{d} E_{v} \approx E^{-2.7}$. Although prompt atmospheric neutrinos are expected theoretically, they have not yet been detected experimentally. The third component arises from a diffuse flux of high energy astrophysical neutrinos (see for example [3]). For the simplest assumption of Fermi-acceleration, the energy spectrum of astrophysical neutrinos is expected to be $\mathrm{d} N_{\text {astro }} / \mathrm{d} E_{v} \approx E^{-2.0}$. A contribution of astrophysical neutrinos to the overall spectrum of $v_{\mu}$ is therefore expected to cause a flattening of the spectrum at high energies. Atmospheric muons are a significant background in neutrino searches and need to be efficiently rejected.

\section{Deconvolution Algorithms}

As it is usually sufficient to obtain the $v_{\mu}$ energy spectrum in a discretized form, deconvolution algorithms generally do not aim at directly solving (1). Instead, they are preceded by a discretizing operation, which transforms (1) into a matrix equation of the form

$$
\vec{f}(E)=A(E, y) \vec{g}(y)
$$

where $\vec{f}(E)$ is the sought after energy spectrum and $\vec{g}(y)$ represents the distribution of an energy-dependent observable. $A(E, y)$ is the so-called response matrix, generally obtained from simulated events. In addition to the physics of neutrino-nucleon interaction, $A(E, y)$ also contains all other effects, such as the propagation of secondary muons through the detection medium and all smearing introduced by the detector itself. Solving (2) is not straight-forward as small eigenvalues in the inverse or pseudoinverse $A^{-1}$ will lead to oscillating non-physical contributions to $\vec{f}(E)$ (see for example [4]).

Several algorithms for solving (2) exist, Iterative Bayesian Unfolding (IBU) [5, 6], Singular Value Decomposition (SVD) [4] and TRUEE [7] being the most common ones. SVD and IBU are also part of the RooUnfold package [8], available for the ROOT [9] data analysis framework. While IBU is based on Bayes' theorem, SVD obtains the inverse $A^{-1}$ via a factorization of the form $A=U S V^{T}$ and the subsequent suppression of small eigenvalues (see [4] 


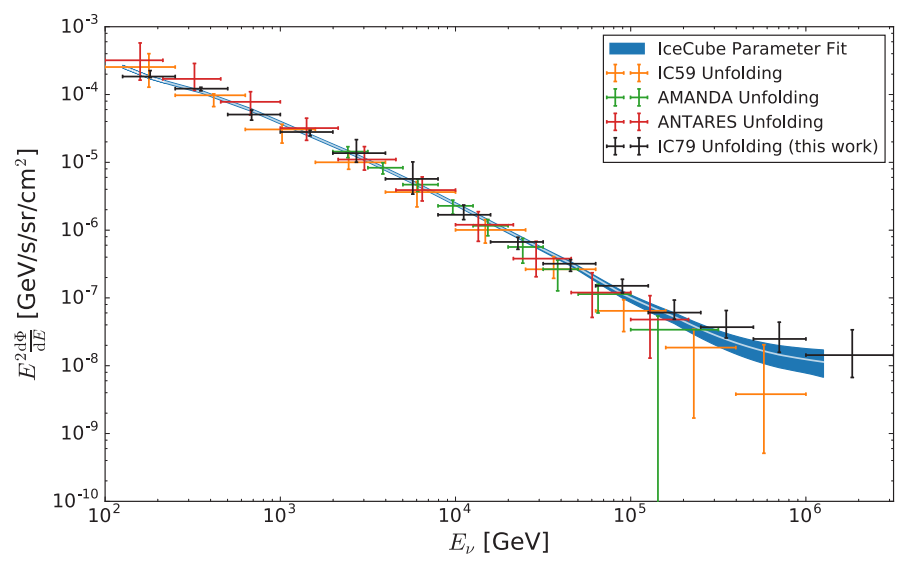

Figure 2. Deconvolution results obtained for IceCube in the 59- (orange) and 79-string configuration (black), compared to an IceCube parameter fit (blue). Energy spectra obtained by ANTARES (red) and AMANDA (green) complete the picture.

for details). TRUEE is based on the RUN-algorithm [10] and uses a maximum likelihood method.

The overall success of a deconvolution depends on the selection of appropriate input variables. These input variables should be well correlated with the energy of the incident neutrino over the entire energy range. Two of the three input variables (the number of photons with small time residuals and the reconstructed energy of the muon) are shown in Fig. 1 In addition to suitable input variables, a deconvolution requires a highly pure sample of neutrino candidates to prevent muons prevent high energy bins (small statistics) from the contamination with atmospheric muons, which might alter the physical interpretation of this particular region of the spectrum. This is due to the steeply falling spectrum of conventional atmospheric neutrinos, which implies that even a small number of atmospheric muons, passing the event selection may mimic the contribution of an additional component to the spectrum at high energies.

\section{Selected Deconvolution Results}

Recent $v_{\mu}$ energy spectra were obtained using IceCube in the 59- [11] and 79-string [12] configuration of the detector. The results are depicted in Fig. 2 and compared to results from ANTARES [13] and AMANDA [14] as well as an IceCube parameter fit [15]. For the data obtained with IC-79 a flattening of the spectrum is observed for neutrino energies exceeding $E_{v}=10^{5} \mathrm{GeV}$. This flattening was found to be consistent with an additional component to the overall flux of neutrinos, which can be attributed to a diffuse flux of high energy neutrinos from astrophysical sources. For IC-59 no such flattening of the spectrum could be observed due to the smaller statistics in the neutrino candidate sample and the larger systematic uncertainties. Furthermore, the IC-79 result extends energy range of the muon neutrino spectrum measured by IceCubce by more than a factor of three, compared to IC-59.

The results for IC-59 and IC-79 were obtained using the TRUEE algorithm. For IC-59 the length of the track insided the detector, the number of unscattered photons and the number of responding DOMs were used as input variables. For IC-79 the number of hit DOMs was replaced by the reconstructed energy of the muons. In both cases, none of the input variables 
was found to have the best correlation with the energy of the neutrino. It was, however, found that the most accurate unfolding result could be obtained using these combinations of variables. For the IC-79 analysis, the correlation of the input variables with the true neutrino energy is depicted in Fig. 1. One finds that all three input variables are reasonably well correlated with the energy of the incident neutrino. In both analyses a high purity sample of neutrinos was obtained using a dedicated event selection chain (see [11] and [12] for details).

\section{Discussion and Outlook}

Although the reconstruction of $v_{\mu}$ energy spectra using data from neutrino telescopes is generally rather challenging, due to the large smearing, accurate results are routinely achieved. Especially in the high energy region, the results are, however, still limited by small statistics, which leads to relatively large statistical uncertainties. This limitation can be overcome in the near future by utilizing multiple years of data taken with IceCube in the final 86-string configuration.

Although the energy spectra obtained in a deconvolution are generally rather precise, the information on individual events is lost during deconvolution. The deconvolution algorithms discussed in Sec. 2, therefore, do not allow the user to investigate which event contributed to what energy bin and how much. The Dortmund Spectrum Estimation Algorithm (DSEA) [16], which is based on machine learning techniques poses a promising alternative for overcoming this limitation in the future.

\section{References}

[1] T.K. Gaisser, R. Engel, E. Resconi, Cosmic Rays and Particle Physics (Cambridge University Press, 2016), ISBN 9780521016469

[2] J. Behringer et al. (Particle Data Group), Phys. Rev. D 86 (2012)

[3] M. G. Aartsen et al. (IceCube Collaboration), Science 342, 1242856 (2013)

[4] A. Höcker, V. Kartvelishvili, Nuclear Instruments and Methods in Physics Research A 372, 469 (1996), hep-ph/9509307

[5] G. D’Agostini, Nuclear Instruments and Methods in Physics Research A 362, 487 (1995)

[6] G. D’Agostini, ArXiv e-prints (2010), 1010.0632

[7] N. Milke, et al., Nuclear Instruments and Methods in Physics Research A 697, 133 (2013), 1209.3218

[8] T. Adye, ArXiv e-prints (2011), 1105.1160

[9] I. Antcheva, M. Ballintijn, B. Bellenot, M. Biskup, R. Brun, N. Buncic, P. Canal, D. Casadei, O. Couet, V. Fine et al., Computer Physics Communications 180, 2499 (2009)

[10] V. Blobel, Regularized unfolding for high-energy physics experiments. Technical Note TN361, OPAL (1996)

[11] M.G. Aartsen, et al., European Physical Journal C 75, 116 (2015), 1409. 4535

[12] M.G. Aartsen et al., European Physical Journal C 77, 692 (2017)

[13] S. Adrián-Martínez, et al., European Physical Journal C 73, 2606 (2013)

[14] R. Abbasi et al., Astropart. Phys. 34, 48 (2010)

[15] M. G. Aartsen et al. (IceCube Collaboration), Phys. Rev. Lett. 115, 081102 (2015)

[16] M. Bunse, N. Piatkowski, T. Ruhe, W. Rhode, K. Morik, Unification of Deconvolution Algorithms for Cherenkov Astronomy, in 5th International Conference on Data Science and Advanced Analytics (DSAA) (IEEE, 2018), pp. 21-30, to appear 